\title{
Usefulness of Modified Alvarado Score in Acute Appendicitis Incorporating Ultrasound
}

\author{
P. Prabhu' ${ }^{1}$ D. Rajiv Raj² \\ 1Department of Surgery, Sri Ramachandra Institute of Higher Education and Research, Chennai, Tamilnadu, India. \\ ${ }^{2}$ Department of Surgery, Sri Ramachandra Institute of Higher Education and Research, Chennai, Tamilnadu, India.
}

\section{ABSTRACT}

\section{BACKGROUND}

Acute appendicitis affects human beings irrespective of age, nationality and region. Acute appendicitis is the most common surgical cause of emergency laparotomy. Considering the difficulties and challenges involved in accurate clinical diagnosis, there is a need for a validated, objective protocol for enabling the diagnosis. This study was carried out to validate the modified Alvarado score (MASS) and correlate with ultrasound and post-operative histopathological examination.

\section{METHODS}

This cohort study was carried out among 100 patients who were suspected with acute appendicitis. Upon clinical evaluation and ultrasound evaluation, Modified Alvarado Score was computed, and patients were taken up for laparotomy/ laparoscopic surgery. The resected specimens were sent for histopathological examination.

\section{RESULTS}

Majority of the participants had a score $>7$ (74\%) of which 51 were males, 21 were females and two were children. Ultrasound detected positive cases in $90 \%$ of the participants, while acute appendicitis by histopathology was present in 54\% of the participants. There was a statistically significant correlation with Modified Alvarado score $(p<0.0001)$. Negative appendicectomy was prevalent in $21 \%$ of the participants.

\section{CONCLUSIONS}

Modified Alvarado Scoring system is ideal for the diagnosis of acute appendicitis since it is simple to use, easy to apply and relies only on history, clinical examination and basic lab investigations.

\section{KEY WORDS}

Acute Appendicitis, Modified Alvarado Score, Laparotomy, Perforation
Corresponding Author:

Dr. D. Rajiv Raj,

Department of Surgery,

Sri Ramachandra Institute of Higher

Education and Research, Chennai,

Tamilnadu, India.

E-mail: drrajiv20012001@yahoo.com

DOI: $10.14260 /$ jemds/2020/108

Financial or Other Competing Interests: None.

How to Cite This Article:

Prabhu P, Raj DR. Usefulness of modified alvarado score in acute appendicitis incorporating ultrasound. J. Evolution Med. Dent. Sci. 2020;9(08):479-482, DOI: 10.14260/jemds/2020/108

Submission 18-12-2019,

Peer Review 30-01-2020,

Acceptance 05-02-2020,

Published 24-02-2020.

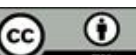




\section{BACKGROUND}

Acute appendicitis affects human beings irrespective of age, nationality and region. In Asia and African countries the incidence is a comparatively lower than those in the Americas and majority of these could be attributed to the diet and lifestyle patterns in these geographic areas. The incidence of appendicitis peaks by 10 years of age and it declines in the geriatric age group. It is highly prevalent in the adolescent age group and in recent years the number of adults in presenting with the acute appendicitis has increased to $6.3 \% .^{[1]}$ several factors have been attributed to the etiology of the acute appendicitis. Among the most common demographic and intrinsic factors, diet has been said to play a major role in determining the incidence of acute appendicitis. Individuals who live on a diet rich in dietary fiber in the form of cellulose are usually immune from the disease but when they adopt life style consisting of increase intake of processed food and refined grains, the incidence rapidly raises.

The diagnosis of acute appendicitis is always being challenging to the surgeons. Acute appendicitis is the most common surgical cause of emergency laparotomy. The disease often progresses to obstructive appendicitis which further leads to perforation and is therefore associated with high rates of morbidity and mortality. In order to combat this, the surgeons are frequently forced to open up the abdomen during emergency, instead of waiting for a definitive diagnosis. The accuracy of clinical examination has been reported to range from $71 \%$ to $91 \%$ depending on the experience of the examiner. However weighing the risk and benefit of certain complications like rupture of appendicitis and other dire consequences, surgeons traditionally prefer to open up the abdomen for acute appendicitis management, thereby accepting a $20 \%$ rate of negative appendectomy and resulting in removal of normal appendicitis.[2]

Considering the difficulties and challenges involved in accurate clinical diagnosis there is a need for a validated, objective protocol for enabling the diagnosis. Although there are several radiological investigations like ultrasound, CT-Scan and laparoscopy, there is always a need for non-invasive and clinically valid method. One of the recently evolved systems of diagnosis has been the Alvarado score which was described and validated in adult surgical practices since 1986. [3] This objective scoring system has a proven to reduce the negative appendicitis rate to 0 to $5 \% .{ }^{[4]}$ The Alvarado score was modified in recent times by M. Kalan, D. Talbat, WJ Cunliffe, A. J. Rich. [5] This scoring system takes in to account variety of symptom complexes including migratory right iliac fossa pain, nausea, vomiting and rebound tenderness in the RIF. The modification carried out by Kalan's and its Cunliffe were mild consisting of minor modifications. However the efficacy and validity of the modified Alvarado scoring system has been seldom explored. The thorough application and validation in the general population will help in implementing the Alvarado score as routine diagnostic tool in clinical practice thereby minimizing the waiting period, and negative appendectomy rates and also ensuring rapid clinical assessment of acute appendectomy.

We wanted to estimate the frequency of acute appendicitis in our tertiary care centre, validate the modified Alvarado score (MASS) and correlate it with post-operative histopathological report.

\section{METHODS}

This prospective cohort study was carried out in the Department of Surgery of our tertiary teaching institution for a period of one year in 2011. All the patients admitted with a suspicion of acute appendicitis namely right iliac fossa pain or tenderness with or without fever during the study period were taken up for the study. A total of 100 patients were included for the study. Patients visiting the hospital with pain abdomen and professionally diagnosed as acquired appendicitis with willingness to undergo surgery were included in the study.

\section{Exclusion Criteria}

1. Diagnoses with pelvic pathology.

2. Appendicular mass.

3. Pregnant females.

4. Patient not willing for surgery.

\section{Ethical Approval and Inform Consent}

Approval was obtained from institutional ethic committee prior to the commencement of the study. Each participant was explained in detail about the study, and informed consent was obtained prior to the commencement of data collection.

\section{Data Collection}

On admission, the patients were subjected to clinical assessment, ultrasound and modified Alvarado score was applied and the score was calculated and noted. (table 1) Ultrasound was done to detect the presence of noncompressible tubular, non-peristaltic, blind ending structure in the right iliac fossa with a diameter $>7 \mathrm{~mm}$. Following a work-up for the surgical intervention the surgery was carried out on the general anaesthesia or spinal anaesthesia. In certain cases laparoscopic appendectomy was also carried out. On identifying the appendicitis it was assessed and resected and the specimen was send for a histopathological examination. The modified Alvarado score was also compared with the histopathological report.

\begin{tabular}{|c|c|c|}
\hline Sl. No. & Alvarado Scoring System Symptom & Score \\
\hline 1 & Migratory RIF pain & 1 \\
\hline 2 & Anorexia & 1 \\
\hline 3 & Nausea and vomiting & 1 \\
\hline 4 & Tenderness over RIF & 2 \\
\hline 5 & Rebound tenderness in RIF & 1 \\
\hline 6 & Fever & 1 \\
\hline 7 & Leucocytosis & 2 \\
\hline \multicolumn{2}{|r|}{ Total Score } & 9 \\
\hline \multirow{3}{*}{ Grading } & $<5$ & Normal \\
\hline & $5-6$ & Mild \\
\hline & $7-9$ & Acute \\
\hline \multicolumn{3}{|c|}{ Table 1. Modified Alvarado Scoring ${ }^{[6]}$} \\
\hline
\end{tabular}

\section{Data Analysis}

Data was entered and analyzed using SPSS Ver. 20 software. The modified Alvarado score was computed as percentages. The correlation with histopathological findings and ultrasound findings carried out using chi square test. A p value $<0.05$ was consider statistically significant.

\section{RESULTS}

This study was carried out among 100 patients admitted for evaluation of various symptoms indicative of acute 
appendicitis. Majority of the participants belonged to 21-30 years of age $(39 \%)$ and were males (64\%). Pain in the right iliac fossa (RIF) was present in all the participants and RIF tenderness was present in $94 \%$ of them. (table 2). Modified Alvarado score was computed and presented in table 3 . Majority of the participants had a score $>7$ (74\%) of which 51 were males, 21 were females and two were children. (table 3)

\begin{tabular}{|c|c|c|c|}
\hline Sl. No. & Characteristics & Frequency & Percentage \\
\hline \multirow{7}{*}{1} & \multicolumn{3}{|c|}{ Age (in Years) } \\
\hline & $\leq 10$ & 2 & 2.0 \\
\hline & $11-20$ & 18 & 18.0 \\
\hline & $21-30$ & 39 & 39.0 \\
\hline & $31-40$ & 23 & 23.0 \\
\hline & $41-50$ & 15 & 15.0 \\
\hline & $>50$ & 3 & 3.0 \\
\hline \multirow{3}{*}{2} & \multicolumn{3}{|c|}{ Sex } \\
\hline & Males & 64 & 64.0 \\
\hline & Females & 36 & 36.0 \\
\hline \multirow{8}{*}{3} & \multicolumn{3}{|c|}{ Clinical Presentation* } \\
\hline & Right Iliac Fossa pain & 100 & 100.0 \\
\hline & Anorexia & 67 & 67.0 \\
\hline & Nausea/Vomiting & 78 & 78.0 \\
\hline & Right Iliac Fossa Tenderness & 94 & 94.0 \\
\hline & Rebound Tenderness & 60 & 60.0 \\
\hline & Fever & 70 & 70.0 \\
\hline & Leukocytosis & 70 & 70.0 \\
\hline \multicolumn{4}{|c|}{ Table 2. Background Characteristics of the Study Participants } \\
\hline \multicolumn{4}{|c|}{${ }^{*} \mathrm{~N}$ will not tally to 100 . } \\
\hline
\end{tabular}

\begin{tabular}{|c|c|c|c|c|}
\hline \multirow{2}{*}{ Sl. No. } & $\begin{array}{c}\text { Modified Alvarado } \\
\text { Score }\end{array}$ & \multicolumn{2}{|c|}{ Adults N=98 n (\%) } & Children N= 2 n \\
\cline { 3 - 5 } & & Males & Females & $(\%)$ \\
\hline $\mathbf{1}$ & $<5$ & $6(6.1)$ & $4(4.1)$ & - \\
\hline $\mathbf{2}$ & $5-6$ & $7(7.1))$ & $9(9.2)$ & - \\
\hline $\mathbf{3}$ & $7-9$ & $51(52.04)$ & $21(21.4)$ & $2(100)$ \\
\hline \multicolumn{4}{|c|}{ Table 3. Findings of Modified Alvarado Score } \\
\hline
\end{tabular}

\begin{tabular}{|c|c|c|c|}
\hline Sl. No. & Diagnostic Finding & Frequency (N=100) & \% \\
\hline \multirow{3}{*}{1.} & \multicolumn{2}{|c|}{ Ultrasonogram } \\
\cline { 2 - 4 } & Positive & 90 & 90 \\
\cline { 2 - 4 } & Negative & 10 & 10 \\
\hline \multirow{4}{*}{2.} & Histopathology & 25 \\
\cline { 2 - 4 } & Lymphoid Hyperplasia & 25 & 54 \\
\cline { 2 - 4 } & Acute Appendicitis & 54 & 6 \\
\cline { 2 - 4 } & Acute Ulcerative & 12 & 3 \\
\cline { 2 - 4 } & Acute Gangrenous & 6 & \\
\hline \multicolumn{3}{|c|}{ Acute Perforative } & 3 \\
\hline
\end{tabular}

\begin{tabular}{|c|c|c|c|c|c|c|c|c|}
\hline \multirow{2}{*}{$\begin{array}{l}\text { Sl. } \\
\text { No. }\end{array}$} & \multirow{2}{*}{\multicolumn{2}{|c|}{$\begin{array}{c}\text { Diagnostic } \\
\text { Test }\end{array}$}} & \multicolumn{3}{|c|}{ MAS Score N (\%) } & \multirow{2}{*}{$\begin{array}{c}\text { Chi } \\
\text { Square } \\
\text { Test }\end{array}$} & \multirow{2}{*}{\multicolumn{2}{|c|}{$\mathbf{p}$}} \\
\hline & & & $\begin{array}{c}<5 \\
N=10\end{array}$ & $\begin{array}{c}5-6 \\
N=16\end{array}$ & $\begin{array}{c}>7 \\
N=74\end{array}$ & & & \\
\hline \multirow{3}{*}{1} & \multicolumn{8}{|c|}{ Ultrasonogram } \\
\hline & & Positive & $9(90)$ & $10(62.5)$ & $71(96)$ & \multirow{2}{*}{155.228} & \multirow{2}{*}{\multicolumn{2}{|c|}{$\begin{array}{c}0.006 \\
\text { (significant) } \\
\end{array}$}} \\
\hline & & Negative & $1(10)$ & $6(37.5)$ & $3(4)$ & & & \\
\hline \multirow{3}{*}{2} & \multicolumn{8}{|c|}{ Histopathology } \\
\hline & Lym & oid Hyperplasia & $10(100)$ & 7 (43.75) & $4(5.4)$ & \multirow{2}{*}{146.275} & \multirow{2}{*}{\multicolumn{2}{|c|}{$\begin{array}{c}0.0001 \\
\text { (significant) }\end{array}$}} \\
\hline & & eappendicitis & $0(0)$ & $9(56.25)$ & $70(94.59)$ & & & \\
\hline \multicolumn{9}{|c|}{ Table 5. Association of MAS with Ultrasonogram and Histopathology } \\
\hline \multicolumn{2}{|c|}{ Sl. No. } & \multicolumn{3}{|c|}{ Negative Appendicectomy } & \multicolumn{3}{|c|}{ Frequency $(\mathrm{N}=21)$} & $(\%)$ \\
\hline \multicolumn{2}{|c|}{1.} & \multicolumn{3}{|c|}{ Male } & \multicolumn{3}{|c|}{11} & 52.38 \\
\hline \multicolumn{2}{|c|}{2.} & \multicolumn{3}{|c|}{ Female } & \multicolumn{3}{|c|}{10} & 47.62 \\
\hline \multirow{2}{*}{\multicolumn{2}{|c|}{3.}} & \multicolumn{3}{|c|}{ Children } & \multicolumn{3}{|c|}{0} & 0 \\
\hline & & able & rcentage & 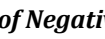 & e Ann & licect & & \\
\hline
\end{tabular}

\begin{tabular}{|c|c|c|c|c|}
\hline Sl. No. & MAS Score & Frequency (N) & Appendicitis & Frequency (\%) \\
\hline \multirow{3}{*}{1.} & \multicolumn{5}{|c|}{ Score $>$ 7 } \\
\cline { 2 - 5 } & Men & 51 & 48 & 96 \\
\cline { 2 - 5 } & Women & 23 & 20 & 90 \\
\hline \multirow{3}{*}{2.} & Men & 7 & 4 & 57.14 \\
\cline { 2 - 5 } & Women & 9 & 5 & 55.55 \\
\hline \multicolumn{5}{|c|}{ Table 7. Validity of MASS } \\
\hline
\end{tabular}

The diagnostic modalities used were ultrasound before the surgery and histopathological examination after the surgery. Ultrasound detected positive in $90 \%$ of the participants, while acute appendicitis by histopathology was present in 54\% of the participants. (table 4). On comparing ultrasound and histopathology findings with MAS score, there was a statistically significant correlation between Modified Alvarado score and the confirmatory tools, indicating validity of Modified Alvarado Score in detecting acute appendicitis. The association was statistically significant $(\mathrm{p}<0.0001)$. (table 5$)$. Negative appendicectomy was prevalent in $21 \%$ of the participants, of which 11 belonged to males and 10 belonged to females. (table 6) The sensitivity of MAS score when it is $>7$ was $96 \%$ in males and $90 \%$ in females. (table 7)

\section{DISCUSSION}

Acute appendicitis remains a common abdominal emergency throughout the world. The diagnosis of acute appendicitis continues to be a challenge due to the variable presentation of the disease and lack of reliable diagnostic test, despite several technological advances in the radiological diagnosis. None of the investigations like USG, CT scan conclusively diagnose appendicitis. When only direct visualization and basic haematological parameters provide a guide towards diagnosis, laparotomy was considered the only choice, despite being invasive in nature. With this background many eminent surgeons and physicians have been adopting different scoring systems in order to decrease negative appendicectomy.

Although there had been considerable advancements in developing a diagnostic protocol for the diagnosis of acute appendicitis over the past several decades, the percentage of normal appendices reported in various series varies from 8 to $33 \% .[7,8]$ Therefore, the advent of clinical scoring system has to an extent eased out the challenge of diagnosis. In the past few years various scores have been developed to aid the diagnosis of acute appendicitis. However, most of these are complex and difficult to implement in the clinical situation. The Alvarado score, is a simple scoring system that can be instituted easily. The present study was undertaken to evaluate the usefulness of Modified Alvarado scoring system in reducing the number of negative appendicectomy and to evaluate the sensitivity of MASS with ultrasound in the diagnosis of acute appendicitis.

The age group in which acute appendicitis occurred commonly was between 11 and 40 years. It is clear that incidence is less in younger and older age groups with peak incidence in $2^{\text {nd }}, 3^{\text {rd }}$ and $4^{\text {th }}$ decade. In the present series the males outnumbered females in the ratio of $1.7: 1$. Pain in the right iliac fossa was the most common presenting symptom and has been observed in all the cases (100\%) in the present series. The classical shifting of pain from umbilical region to RIF was seen in majority cases. Next common symptoms observed were nausea/ vomiting in $78 \%$ of cases and anorexia in $67 \%$ of cases. Fever was of low grade with corresponding rise in pulse rate and was present in $70 \%$ of cases. Majority of the patients presented within $24 \mathrm{hrs}$. after the onset of pain, with most of them presenting between 12-24 hrs. of onset of pain. On clinical examination, tenderness at McBurney's point was the commonest sign (94\%). Rebound tenderness was present in $60 \%$. Abdominal rigidity in $8 \%$ was due to perforated appendix or gangrenous appendix.

In the present study the Total Leukocyte Count was increased in $70 \%$. For assessment, the patients were 
categorized into 3 groups namely, male, female and children. Out of 100 cases studied, 64 were male, 36 were female and 2 were children ( $<12$ yrs). Majority (98\%) of the participants underwent laparoscopic Appendicectomy and remaining two participants underwent open appendicectomy for nonmedical reasons. In this study, $79.6 \%$ of the males and $63.8 \%$ of the females had a score of $>7-9$. All the 2 children had score $>7-9$. Acute appendicitis was present in $34 \%$ of the total study participants. In our series a score of 7-9 using modified Alvarado system had a total sensitivity of $94.59 \%$. Our study findings were consistent with other study findings. [9] When compared with other study it is evident that Modified Alvarado scoring system more sensitivity, thereby proving that it can be used as a complementary method in diagnosing acute appendicitis.

With regards to negative appendicectomy, increased proportion (43.75\%) of negative appendicectomy is noticed for the Modified Alvarado Score 5-6 and significantly decreased proportion (5.40\%) negative appendicectomy is noticed for the modified Alvarado score 7-9 ( $p$ value-0.0001). In our series negative appendicectomy rate in females with score 5-6 was $44.44 \%$ and with score $7-9$ was $8.69 \%$. Men with score 5-6 had negative appendicectomy rate of $42.85 \%$ and with score 7-9 had negative appendicectomy rate of $3.92 \%$. Hence in the overall females (27.8\%) had more negative appendicectomy rate compared to males (17.18\%). This may be justified by the fact that females are prone for other diseases like pelvic inflammatory diseases, especially in the reproductive age group. Since intra-abdominal infection in females, particularly lower abdomen, can be quite challenging in terms of diagnosis, it is difficult to differentiate acute appendicitis from gynecological conditions like twisted ovarian cyst and PID.

The modified Alvarado score $>7$ has got more sensitivity (94.59\%) and diagnostic accuracy for appendicitis. Those patients who scored $<5$ did not require subsequent laparotomy, indicating the usefulness of the system in ruling out acute appendicitis. This indicates that by particularly adopting this system, negative laparotomies can be reduced by a figure of $21 \%$. While comparing USG with MASS score $>7$ has more sensitivity (95.59\%) thereby increasing diagnostic accuracy ( $p$ value 0.006). Ultrasonography, in this study detected $86 \%$ of cases of histologically proven cases of appendicitis. This study showed ultrasound to have sensitivity of $86 \%$, specificity of $75 \%$ and positive predictive value of about $86 \%$ ( $\mathrm{p}$ value 0.0001 ). Ultrasound remains to be operator dependent. This study shows along with clinical examination and in doubtful cases to rule out other conditions causing pain, ultrasound proves to be a valuable tool.

\section{CONCLUSIONS}

It is clear that incidence of acute appendicitis is less in younger and older age groups with peak incidence in $2^{\text {nd }}, 3^{\text {rd }}$ and $4^{\text {th }}$ decade. Our study correlated the Modified Alvarado score with the histopathological reports of the appendix and found that there is $\mathbf{9 4 \%}$ sensitivity. Modified Alvarado Scoring system is ideal for the diagnosis of acute appendicitis since it is simple to use, easy to apply and relies only on history, clinical examination and basic lab investigations. It is cost-effective and can be used by junior residents in all district general hospitals with basic lab facilities. Modified Alvarado Score complimented with ultrasound, increased the diagnostic accuracy resulting in avoiding negative laparotomy. Based on our study, it may be recommended that emergency appendectomy can be carried out in all patients whose clinical score is more than 7. Modified Alvarado scoring system significantly reduces the number of negative laparotomies without increasing overall rate of appendicular perforation.

\section{REFERENCES}

[1] Lohar HP, Calcuttawala MAS, Nirhale DS, et al. Epidemiological aspects of appendicitis in a rural set up. Med J DY Patil Univ 2014;7 (6):753-7.

[2] John H, Neff U, Kelemen M. Appendicitis diagnosis today: clinical and ultrasonic deductions. World J Surg 1993;17 (2):243-9.

[3] Jones PF. Suspected acute appendicitis: trends in management over 30 years. Br J Surg 2001;88 (12):1570 7.

[4] Lee SL, Walsh AJ, Ho HS. Computed tomography and Ultrasonography do not improve and may delay the diagnosis and treatment of acute appendicitis. Arch Surg 2001;136 (5):556-61.

[5] Kakande I, Kavuma J, Kampala E. Alvarado Score. East Afr Med J 1978;55 (4):172-6.

[6] Alvarado A. A practical score for the early diagnosis of acute appendicitis. Ann Emerg Med 1986;15 (5):557-64.

[7] Fitz RH. Perforating inflammation of the veriform appendix: with special reference to its early diagnosis and treatment. Am J Med Sci 1886;92:321-46.

[8] Teicher I, Landa B, Cohen M, et al. Scoring system to aid in diagnosis of appendicitis. Ann Surg 1983;198 (6):753-9.

[9] Kalan M, Rich AJ, Talbot D, etal. Evaluation of the modified Alvarado score in the diagnosis of acute appendicitis: a prospective study. Ann R Coll Surg Engl 1994;76 (6):4189. 